\title{
Erratum to: Significant treatment effect of adjunct music therapy to standard treatment on the positive, negative, and mood symptoms of schizophrenic patients: a meta-analysis
}

\author{
Ping-Tao Tseng ${ }^{1{ }^{*}+}$, Yen-Wen Chen ${ }^{2+}$, Pao-Yen Lin ${ }^{3,4}$, Kun-Yu Tu' ${ }^{1}$, Hung-Yu Wang ${ }^{1}$, Yu-Shian Cheng ${ }^{1}$, \\ Yi-Chung Chang ${ }^{1}$, Chih-Hua Chang ${ }^{1}$, Weilun Chung ${ }^{1}$ and Ching-Kuan Wu ${ }^{1}$
}

In article "Significant treatment effect of adjunct music therapy to standard treatment on the positive, negative, and mood symptoms of schizophrenic patients: a metaanalysis [1]", some values of Hedges' g in main results of the meta-analysis might mislead the readers' interpretation of our results. Different values of Hedges' g may derive from different methods of standardization when using Comprehensive Meta-analysis software. The main results of significance in current study remained significant. By using different method of standardization, we found that the main treatment effect of adjunct music therapy in schizophrenia was significantly larger than those without adjunct music therapy (Hedges' $\mathrm{g}=0.596$, $95 \% \mathrm{CI}=0.350-0.842, p<0.001)$. At the same time, the treatment effect of adjunct music therapy in schizophrenia remained significantly larger than those without adjunct music therapy in scores of positive symptoms, negative symptoms, and mood symptoms (Hedges' $\mathrm{g}=0.483,95 \%$ $\mathrm{CI}=0.053-0.913, p=0.028$; Hedges' $\mathrm{g}=0.673,95 \% \mathrm{CI}=$ $0.385-0.961, p<0.001$; Hedges' $\mathrm{g}=0.677,95 \% \mathrm{CI}=0.434$ $0.919, p<0.001$, separately).

\footnotetext{
* Correspondence: ducktseng@gmail.com

${ }^{\dagger}$ Equal contributors

${ }^{1}$ Department of Psychiatry, Tsyr-Huey Mental Hospital, Kaohsiung Jen-Ai's

Home, Taiwan, No.509, Fengping 1st Rd., Daliao Dist, Kaohsiung 831, Taiwan

Full list of author information is available at the end of the article
} 


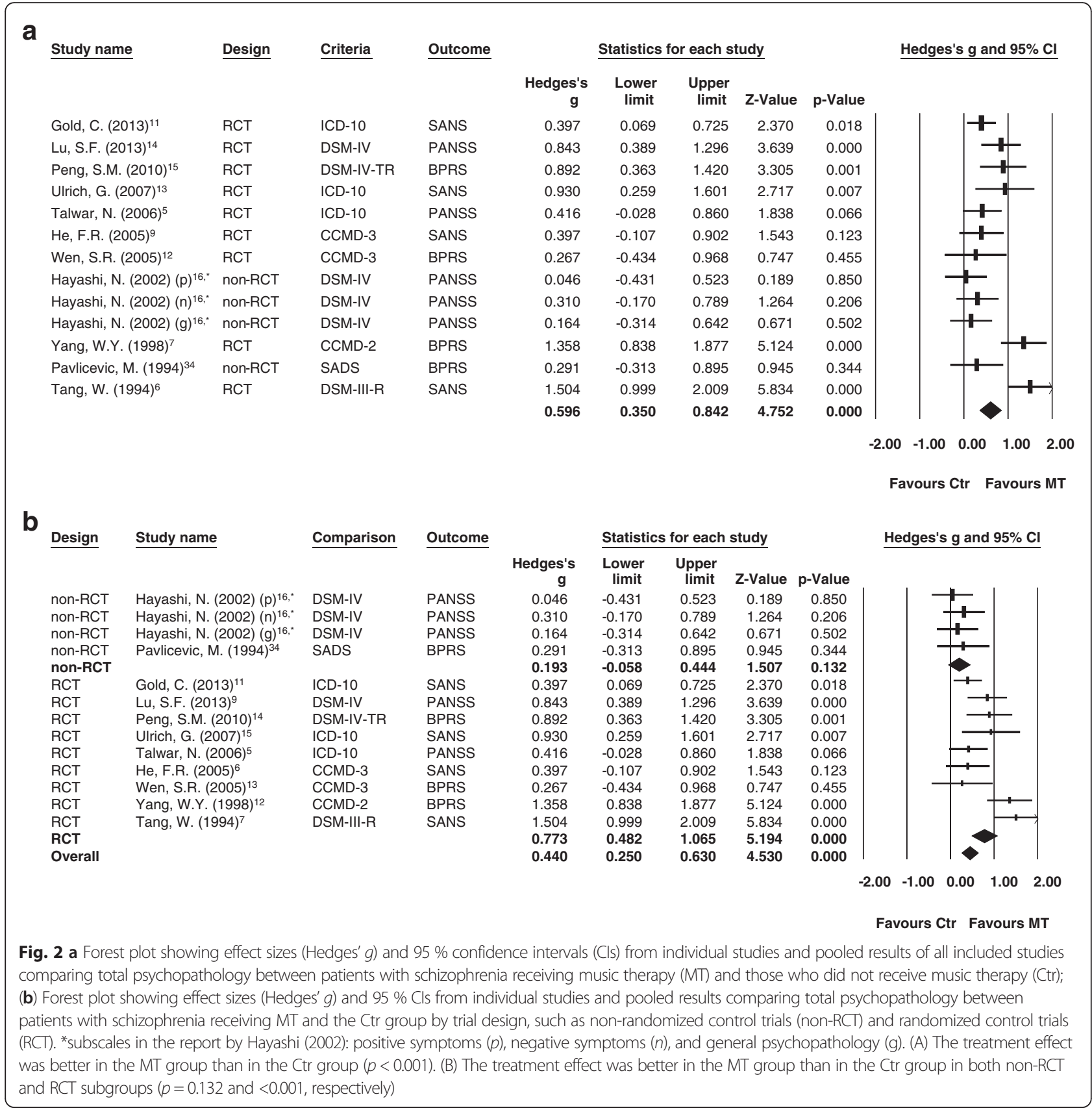




\begin{tabular}{|c|c|c|c|c|c|c|c|c|}
\hline \multirow{2}{*}{$u$} & Study name & Comparison & Outcome & \multicolumn{4}{|c|}{ Statistics for each study } & \multirow[t]{2}{*}{ Hedges's $\mathrm{g}$ and $95 \% \mathrm{Cl}$} \\
\hline & & & & \multirow{2}{*}{$\begin{array}{c}\text { Hedges's } \\
\text { g }\end{array}$} & \multirow{2}{*}{$\begin{array}{l}\text { Lower } \\
\text { limit }\end{array}$} & \multicolumn{2}{|l|}{ Upper } & \\
\hline & & & & & & limit & Z-Value & p-Value \\
\hline & Lu, S.F. $(2013)^{14}$ & DSM-IV & PANSS & 0.849 & 0.395 & 1.303 & 3.666 & 0.000 \\
\hline & Peng, S.M. $(2010)^{15}$ & DSM-IV-TR & BPRS & 0.892 & 0.363 & 1.420 & 3.305 & 0.001 \\
\hline & Talwar, N. $(2006)^{5}$ & ICD-10 & PANSS & 0.175 & -0.265 & 0.615 & 0.779 & 0.436 \\
\hline & Hayashi, N. $(2002)^{16}$ & DSM-IV & PANSS & 0.046 & -0.431 & 0.523 & 0.189 & 0.850 \\
\hline & & & & \multirow[t]{2}{*}{0.483} & \multirow[t]{2}{*}{0.053} & \multirow[t]{2}{*}{0.913} & \multirow[t]{2}{*}{2.201} & 0.028 \\
\hline & & & & & & & & $\begin{array}{lll}-2.00 & -1.00 & 0.00\end{array}$ \\
\hline & & \multicolumn{6}{|c|}{ Positive symptoms } & Favours Ctr Favours MT \\
\hline \multirow[t]{15}{*}{$\mathbf{D}$} & Study name & Criteria & Outcome & \multicolumn{4}{|c|}{$\underline{\text { Statistics for each study }}$} & Hedges's $\mathrm{g}$ and $95 \% \mathrm{Cl}$ \\
\hline & & & & $\begin{array}{c}\text { Hedges's } \\
\text { g }\end{array}$ & $\begin{array}{c}\text { Lower } \\
\text { limit }\end{array}$ & $\begin{array}{r}\text { Upper } \\
\text { limit }\end{array}$ & Z-Value & $\mathrm{p}$-Value \\
\hline & Gold, C. (2013) $)^{11}$ & ICD-10 & SANS & 0.397 & 0.069 & 0.725 & 2.370 & 0.018 \\
\hline & Lu, S.F. $(2013)^{14}$ & DSM-IV & PANSS & 0.535 & 0.093 & 0.978 & 2.371 & 0.018 \\
\hline & Peng, S.M. $(2010)^{15}$ & DSM-IV-TR & BPRS & 0.667 & 0.149 & 1.185 & 2.524 & 0.012 \\
\hline & Ulrich, G. $(2007)^{13}$ & ICD-10 & SANS & 0.930 & 0.259 & 1.601 & 2.717 & 0.007 \\
\hline & Talwar, N. $(2006)^{5}$ & ICD-10 & PANSS & 0.503 & 0.058 & 0.949 & 2.213 & 0.027 \\
\hline & He, F.R. $(2005)^{9}$ & CCMD-3 & SANS & 0.397 & -0.107 & 0.902 & 1.543 & 0.123 \\
\hline & Hayashi, N. (2002) ${ }^{16}$ & DSM-IV & PANSS & 0.310 & -0.170 & 0.789 & 1.264 & 0.206 \\
\hline & Yang, W.Y. $(1998)^{7}$ & CCMD-2 & SANS & 1.522 & 0.991 & 2.054 & 5.612 & 0.000 \\
\hline & Pavlicevic, M. (1994) & SADS & SANS & 0.069 & -0.531 & 0.670 & 0.227 & 0.821 \\
\hline & \multirow[t]{3}{*}{ Tang, W. (1994) ${ }^{6}$} & DSM-III-R & SANS & 1.504 & 0.999 & 2.009 & 5.834 & 0.000 \\
\hline & & & & 0.673 & 0.385 & 0.961 & 4.581 & 0.000 \\
\hline & & & & & & & & $\begin{array}{lllll}-2.00 & -1.00 & 0.00 & 1.00 & 2.00\end{array}$ \\
\hline & & & & tive sym & toms & & & Favours Ctr $\quad$ Favours MT \\
\hline \multirow[t]{8}{*}{ C } & Study name & Criteria & Outcome & \multicolumn{4}{|c|}{ Statistics for each study } & Hedges's $\mathrm{g}$ and $95 \% \mathrm{Cl}$ \\
\hline & & & & $\begin{array}{c}\text { Hedges's } \\
\text { g }\end{array}$ & $\begin{array}{l}\text { Lower } \\
\text { limit }\end{array}$ & $\begin{array}{c}\text { Upper } \\
\text { limit }\end{array}$ & Z-Value & $\mathrm{p}$-Value \\
\hline & Lu, S.F. $(2013)^{14}$ & DSM-IV & CDSS & 0.958 & 0.499 & 1.418 & 4.090 & 0.000 \\
\hline & Peng, S.M. $(2010)^{15}$ & DSM-IV-TR & BPRS & 0.675 & 0.157 & 1.194 & 2.555 & 0.011 \\
\hline & Li, Y.M. $(2007)^{10}$ & CCMD-3 & SDS & 0.684 & 0.170 & 1.199 & 2.609 & 0.009 \\
\hline & Wen, S.R. $(2005)^{12}$ & CCMD-3 & HAM-D & 0.522 & -0.188 & 1.232 & 1.441 & 0.150 \\
\hline & Pavlicevic, M. (1994) $)^{34}$ & SADS & HAM-D & 0.291 & -0.313 & 0.895 & 0.945 & 0.344 \\
\hline & & & & 0.677 & 0.434 & 0.919 & 5.463 & 0.000 \\
\hline & & & & & & & & $\begin{array}{lllll}-2.00 & -1.00 & 0.00 & 1.00 & 2.00\end{array}$ \\
\hline \multicolumn{8}{|c|}{ Mood symptoms } & Favours Ctr Favours MT \\
\hline \multicolumn{9}{|c|}{$\begin{array}{l}\text { Fig. } 3 \text { Forest plot showing effect sizes (Hedges' } g \text { ) and } 95 \% \text { confident intervals (Cls) from individual studies and pooled results comparing (a) positive } \\
\text { symptoms, (b) negative symptoms, and (c) mood symptoms between schizophrenic patients who received music therapy (MT) and those who did not } \\
\text { (Ctr). (A) The treatment effect was better in the MT group compared to the Ctr group in subscales of positive symptoms }(p=0.028) \text {. (B) The treatment } \\
\text { effect was better in the MT group compared to the Ctr group in subscales of negative symptoms ( } p<0.001 \text { ). (C) The treatment effect was better in the MT } \\
\text { group compared to the Ctr group in subscales of mood symptoms }(p<0.001)\end{array}$} \\
\hline
\end{tabular}




\section{Author details}

'Department of Psychiatry, Tsyr-Huey Mental Hospital, Kaohsiung Jen-Ai's

Home, Taiwan, No.509, Fengping 1st Rd., Daliao Dist, Kaohsiung 831, Taiwan. ${ }^{2}$ Department of Neurology, E-Da Hospital, Kaohsiung, Taiwan. ${ }^{3}$ Department of Psychiatry, Kaohsiung Chang Gung Memorial Hospital and Chang Gung University College of Medicine, Kaohsiung, Taiwan. ${ }^{4}$ Center for Translational Research in Biomedical Sciences, Kaohsiung Chang Gung Memorial Hospital, Kaohsiung, Taiwan.

Received: 20 April 2016 Accepted: 4 May 2016

Published online: 17 May 2016

\section{Reference}

1. Tseng PT, Chen YW, Lin PY, Tu KY, Wang HY, Cheng YS, et al. Significant treatment effect of adjunct music therapy to standard treatment on the positive, negative, and mood symptoms of schizophrenic patients: a metaanalysis. BMC Psychiatry. 2016;16(1):16.

Submit your next manuscript to BioMed Central and we will help you at every step:

- We accept pre-submission inquiries

- Our selector tool helps you to find the most relevant journal

- We provide round the clock customer support

- Convenient online submission

- Thorough peer review

- Inclusion in PubMed and all major indexing services

- Maximum visibility for your research

Submit your manuscript at www.biomedcentral.com/submit
Biomed Central 